\title{
BMJ Open Association of body flexibility and carotid atherosclerosis in Japanese middle-aged men: a cross-sectional study
}

\author{
Masataka Suwa, ${ }^{1}$ Takayuki Imoto, ${ }^{1}$ Akira Kida, ${ }^{1}$ Takashi Yokochi, ${ }^{1,2}$ \\ Mitsunori Iwase, ${ }^{3}$ Kenji Kozawa ${ }^{1}$
}

To cite: Suwa M, Imoto T, Kida A, et al. Association of body flexibility and carotid atherosclerosis in Japanese middle-aged men: a crosssectional study. BMJ Open 2018;8:e019370. doi:10.1136/ bmjopen-2017-019370

- Prepublication history for this paper is available online. To view these files, please visit the journal online (http://dx.doi org/10.1136/bmjopen-2017019370).

Received 31 August 2017 Accepted 15 November 2017

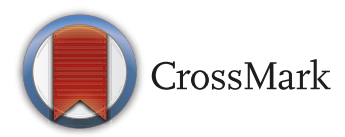

${ }^{1}$ Health Support Center WELPO, Toyota Motor Corporation, Toyota, Japan

${ }^{2}$ Midtown Clinic Meieki, Nagoya, Japan

${ }^{3}$ Toyota Memorial Hospital,

Toyota, Japan

Correspondence to Dr Masataka Suwa; masataka_suwa@mail.toyota. co.jp

\section{ABSTRACT}

Objective This study examined the associations of body flexibility with carotid arterial remodelling, including intima-media thickness (IMT) and plaque formation in middle-aged men.

Methods The subjects of this cross-sectional study included 1354 Japanese men aged 35-59 years without histories of stroke or cardiac diseases. The arm extensibility test, which can estimate flexibility of the upper extremity (composed of shoulder external rotation and forearm supination), and the sit-and-reach test were performed. Common carotid IMT and plaque formation $(\geq 1.1 \mathrm{~mm})$ were estimated by ultrasound.

Results The proportion of subjects who fully completed the arm extensibility test was $55.0 \%$, and who had plaques in the common carotid artery was $37.8 \%$. IMT was associated with poor arm extensibility ( $\beta=-0.073,95 \% \mathrm{Cl}-0.02224$ to $=0.00041, \mathrm{P}=0.004)$, while plaque formation was associated with poor sit-andreach (OR 0.98579, 95\% $\mathrm{Cl} 0.97257$ to $0.99919, \mathrm{P}=0.038$ ) after adjustment by all covariates.

Conclusions This study demonstrated that poor upper extremity and trunk flexibility were associated with characteristics of early onset of atherosclerosis. Furthermore, these associations were independent of covariates such as age, blood pressure, blood lipids glucose levels and abdominal fat accumulation, handgrip strength and lifestyle, including sleeping, drinking, exercise and smoking habits. Poor flexibility may reflect subclinical atherosclerosis in middle-aged men.

\section{INTRODUCTION}

Atherosclerosis is an important cause of cerebrovascular and cardiovascular events. ${ }^{1}$ Carotid arterial remodelling such as progressing carotid intima-media thickness (IMT) and plaque formation are used as ultrasound indicators of carotid atherosclerosis. ${ }^{24}$ Increased carotid IMT may represent the early phase of carotid atherosclerosis, while the presence of carotid plaques may reflect later and advanced phases of the atherosclerotic process. ${ }^{56}$ Carotid IMT and plaques are objective morphological indicators of
Strengths and limitations of this study

- Simple, easy, safe and inexpensive tests of body flexibility such as arm extensibility (upper extremity flexibility) and sit-and-reach (trunk flexibility) tests were adopted for evaluating body flexibility.

- The study is limited by the cross-sectional nature and characteristics of subjects (middle-aged Japanese men employed by Toyota Motor Corporation).

biological processes of atherosclerosis, and were proposed as surrogate markers of ischaemic stroke and cardiovascular diseases. ${ }^{1}$

There is increasing evidence that poor physical fitness, including cardiorespiratory fitness and muscular strength, are the risk factors for mortality and chronic diseases including type 2 diabetes mellitus, cardiovascular diseases, stroke, cancer and dementia. ${ }^{78}$ Flexibility, the ability to move a joint through its complete range of motion, is also a component of physical fitness. Flexibility is dependent on distensibility of the joint capsule, muscle viscosity and compliance of the ligaments and tendons. ${ }^{9}$

Recent studies have shown that trunk flexibility measured by sit-and-reach test is inversely associated with arterial stiffness, including the pulse wave velocity $(\mathrm{PWV})^{1011}$ and Cardio-Ankle Vascular Index (CAVI). ${ }^{11}$ This association is stronger in men $(\beta=-0.142)$ than women $(\beta=-0.044) .{ }^{11}$ In men, the results vary between young $(<40$ years $)$, middle (40-59 years) and older ( $\geq 60$ years) ages, and consistent results are reported only in middle-aged men. ${ }^{10} 11$ In young men, both CAVI- and brachial-ankle PWV-estimated arterial stiffness were negatively associated with sit-and-reach, ${ }^{11}$ whereas another study ${ }^{10}$ demonstrated no association of brachial-ankle or aortic PWVs in men or women. In middleaged men, significant negative associations 


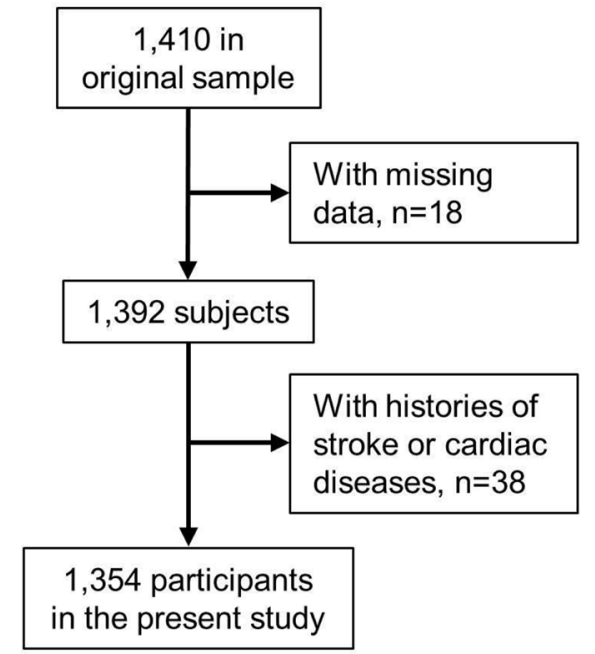

Figure 1 Flow diagram of participation in this study.

were reported between arterial stiffness measured by any methods and sit-and-reach, ${ }^{10}{ }^{11}$ while in older men the findings are inconsistent. ${ }^{10}{ }^{11}$ However, associations between body flexibility and arterial morphological characteristics remain unknown. Nevertheless, carotid IMT is reported to be associated with PWV and CAVI values. ${ }^{12}$ Collectively these findings suggest that flexibility may be associated with carotid atherosclerotic processes especially in middle-aged men.

In the current study, we examined the hypothesis that poor flexibility is related to subclinical atherosclerosis, including increasing carotid IMT and presence of plaque in middle-aged men.

\section{METHODS}

\section{Ethical approval of the study protocol}

The study was conducted in accordance with the Declaration of Helsinki. All individuals provided written consent to participate in this study.

\section{Study design and population}

The present study was carried out as part of the baseline survey of the Toyota Motor Corporation Physical Activity and Fitness Study, and was conducted from October 2015 to January 2016. ${ }^{13}$ Subjects in the baseline study were 1410 Japanese men, aged 35-59 years, who were working for the Toyota Motor Corporation (Aichi, Japan). Eighteen individuals were excluded because of incomplete data, while a further 38 individuals with histories of stroke or cardiac diseases were excluded. A final 1354 subjects were included in the present study (figure 1). All subjects received annual medical examinations in accordance with the Industrial Safety and Health Law of Japan. Employees were required by law to participate, and all clinical data were supplied as medical examination data.

\section{Medical examinations}

After an overnight fast of $\geq 11$ hour, subjects underwent measurement of anthropometry, systolic blood pressure (SBP), diastolic blood pressure (DBP), blood chemistry analyses, ultrasound examination and CT. Pulse pressure was calculated reducing DBP from SBP. Height and body mass were measured using an automated measuring instrument (BF-220; Tanita, Tokyo, Japan), from which the body mass index was calculated. Percentage of body fat was determined by biochemical impedance (BF-220; Tanita). Waist circumference was measured at the level of the umbilicus in a standing position while breathing normally (at the end of expiration while breathing gently). Blood samples were drawn from the antecubital vein from seated subjects.

Assessment of carotid IMT and plaques by ultrasound images All estimations of carotid IMT and plaque were performed by well-trained clinical technologists who were blinded to other clinical information. Common carotid IMT and plaque presence were estimated with an Aplio 500 ultrasound device (TUS-A500; Toshiba Medical Systems, Otawara, Tochigi, Japan) by manual method using a 7.5 MHz probe frequency. All subjects were examined in the supine position. A long axial section of the carotid artery was probed to show the common carotid artery on both sides to measure IMT and to detect plaques. IMT was measured in the far wall at $\sim 20 \mathrm{~mm}$ from the carotid bifurcation using recorded images of the carotid artery. If plaques were observed around the point, IMT was determined at a point avoiding the plaques. Furthermore, if a defective image of the far wall was observed, IMT was determined in the near wall. The mean IMT of each side was used for analysis. Plaques were defined as elevated lesions with a maximum thickness of $\geq 1.1 \mathrm{~mm}$, and having a point of inflection on the surface of the intima-media complex as previously reported. ${ }^{14}$ The plaque was measured on a short axial section. If multiple plaques were observed, the maximum value was used for analysis.

\section{Assessment of adipose tissues by CT}

CT of the abdomen was performed at the end of the expiratory phase using an Aquillion system (Toshiba Medical Systems, Tochigi, Japan). The umbilicus was assessed for areas of visceral and subcutaneous fat, which were measured in accordance with the guidelines for obesity treatment set by the Japan Society for the Study of Obesity. ${ }^{15}$ Modified measurement levels were employed if subjects possessed a clearly low umbilical body type. Image analysis software (SlimVision V.4.0; Cybernet Systems, Tokyo, Japan) was used at an attenuation range from -70 to -160 Hounsfield units to quantify abdominal areas of adipose tissue. The subcutaneous fat area (SFA) was defined as fat superficial to the abdominal and back muscles. The visceral fat area (VFA) was defined as intra-abdominal fat bound by the parietal peritoneum or fascia transversalis. 


\section{Biochemical assays}

Fasting blood glucose (FBG) levels were measured by the hexokinase-glucose-6-phosphate dehydrogenase method (Eiken Chemicals, Tokyo, Japan). Concentrations of glycated haemoglobin were measured by high-performance liquid chromatography. Concentrations of triglycerides (TGs) were measured by enzymatic colorimetric analyses (standard methods set by the Japan Society of Clinical Chemistry and Reference Material Institute for Clinical Chemistry Standards). Total cholesterol levels were measured by the cholesterol oxidase-peroxidase method. Levels of high-density lipoprotein cholesterol (HDL-C) and low-density lipoprotein cholesterol (LDL-C) were measured using the chemically modified enzyme method (Metaboredo HDL-C, Metaboredo LDL-C; Kyowa Medex, Tokyo, Japan).

\section{Lifestyle estimation}

A self-administered questionnaire was administered to assess $\geq 30 \mathrm{~min}$ of exercise (none, 1 ; once/week, 2; 2-6 times/week, 3; every day, 4), smoking (never, 1; former, 2; current, 3), alcohol consumption (none, 1; sometimes,
2; 3 times/week, 3; every day, 4) and sleeping time (hour/day).

\section{Measurements of flexibility and handgrip strength}

We estimated physical fitness levels, including upper extremity and trunk flexibility and handgrip strength. All estimations were performed by independent experts in exercise testing who were blinded to other clinical information collected in this study.

Upper extremity flexibility was determined by the arm extensibility test, which can estimate the ranges of shoulder external rotation and forearm supination. Details of the arm extensibility test are shown in figure 2. In brief, the first trial estimated whether an individual was able to fully extend both elbows while interlocking their hands in the right-arm crossing over position. Next, a similar trial of the left-arm crossing over position was performed. If the individual fully extended both elbows in both trials, the subject was judged as 'complete'. We confirmed the reproducibility of this test. The per cent agreements between observers 1 and $2(n=58)$ and between observers 1 and $3(\mathrm{n}=76)$ were $98.3 \%$ and
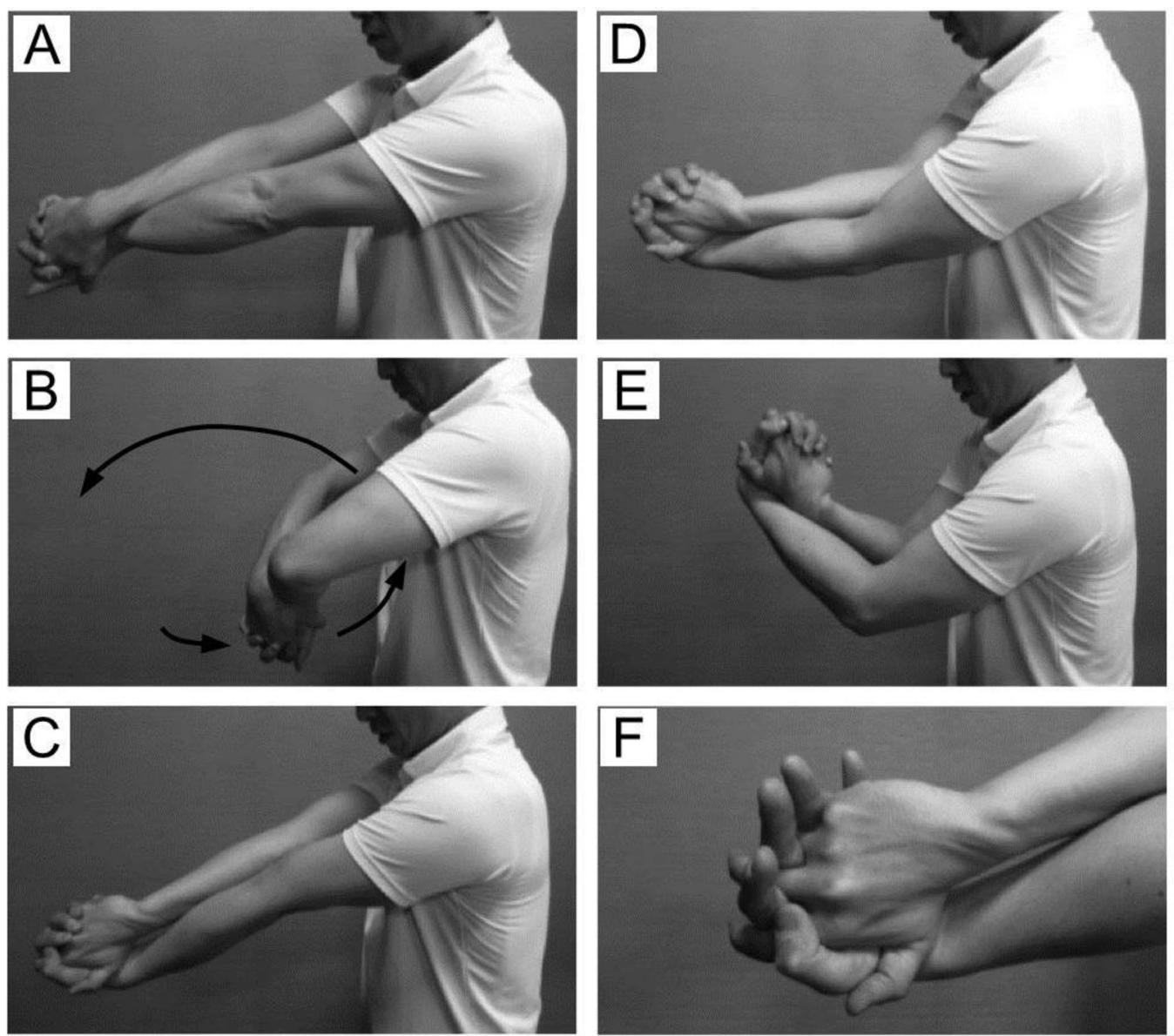

Figure 2 Arm extensibility test (right-arm crossing over position). (A) Beginning position. The right arm is crossed over the left arm in front of subjects' body, with interlocking hands. (B) The subjects pass their interlocked hands through a loop made by the arms, and extend their elbows as far as possible. (C) Subjects who fully extend their elbows with their hands interlocked are judged as 'complete in right-arm crossing over position'. (D and E) Subjects that cannot fully extend their elbows are judged as 'incomplete'. (F) Subjects that cannot keep their hands interlocked are judged as 'incomplete'. 
$97.4 \%$, respectively, and the $\kappa$ coefficients were 0.96 and 0.94 , respectively.

Trunk flexibility was assessed by a sit-and-reach test using a device (T.K.K. 5412; Takei Scientific Instruments, Niigata, Japan). Subjects sat on the floor with their hips, back and occipital region of the head touching a wall, and their legs held straight at $90^{\circ}$ in front of the upper body. The zero point of the device was set in this position. Subjects then slowly bent forward and as far as possible while pushing the device with both hands, and the moving distance of the device was measured. Measurements were performed two times, and the maximum value was used in final analyses.

Handgrip strength was measured using a handgrip dynamometer (T.K.K. 5401; Takei Scientific Instruments). Measurements were performed in duplicate in each hand, and the mean maximum force of each hand was used in final analyses.

\section{Statistical analysis}

Data are shown as mean $\pm \mathrm{SD}$. An unpaired t-test was used to compare mean values between subjects who did not complete and those who completed in both arm positions in the arm extensibility test, and between subjects who had plaques in the common carotid artery and those who did not. Arm extensibility (incomplete or complete), presence of plaque (yes or no) and lifestyle differences, including $\geq 30 \mathrm{~min}$ of exercise (none, or more than once/week), alcohol consumption (none, or more than sometimes) and cigarette smoking (never, former or current) were compared using the $\chi^{2}$ test.

Multiple linear regression models were performed to estimate the association of IMT with arm extensibility or sit-and-reach. Three models were used to assess these associations: model 1 was crude, model 2 was adjusted for age and height and model 3 was adjusted for model 2 covariates plus SFA, VFA, DBP, pulse pressure, TG, HDL-C, LDL-C, FBG, sleeping time, exercise habit, drinking habit, smoking habit, handgrip strength and sit-and-reach (arm extensibility analysis) or arm extensibility (sit-and-reach analysis).

The relationships between the presence of plaque and arm extensibility or sit-and-reach were analysed using multiple logistic regression models. The same three models used for multiple linear regression analyses for estimating IMT were used.

All statistical analyses were performed using statistical software (SPSS V.23.0; IBM, Armonk, New York, USA). Differences were considered statistically significant if $\mathrm{P}<0.05$.

\section{RESULTS}

\section{Anthropometry and medical and fitness examinations}

Anthropometry data and medical and fitness examinations in the study subjects are shown in table 1. A total of 745 subjects $(55.0 \%)$ fully completed the arm extensibility test, and 512 subjects $(37.8 \%)$ had a common carotid plaque. Mean IMT $(-6.9 \%)$ and the proportion of subjects with a plaque $(-26.2 \%)$ were significantly lower, while the sit-and-reach test distance $(+16.0 \%)$ was significantly higher, in subjects who completed the arm extensibility test compared with subjects who did not complete. Furthermore, the proportion of subjects who fully completed the arm extensibility test $(-20.3 \%)$ and the distance of the sit-and-reach test $(-4.5 \%)$ were significantly lower, while the mean IMT was significantly higher, in subjects who had a plaque compared with those without plaques.

\section{Association between body flexibility indicators}

The association of the sit-and-reach test with the arm extensibility test was estimated using linear regression models. Sit-and-reach was inversely associated with arm extensibility in both non-adjusted $(\beta=-0.190, \mathrm{P}<0.001)$ and age-adjusted $(\beta=-0.086, \mathrm{P}=0.001)$ models.

\section{Association of IMT and body flexibility}

The associations of IMT with the arm extensibility or sit-and-reach tests using multivariate linear regression models are shown in table 2. Overall arm extensibility was inversely associated with IMT, whereas the sit-and-reach was unrelated to IMT.

\section{Association of plaque formation and body flexibility}

The associations of presence of plaques with the arm extensibility or sit-and-reach tests using multivariate logistic regression models are shown in table 3. For arm extensibility, there was a significant positive association observed in model 1 , which was no observed after adjusting for covariates. The sit-and-reach test was positively associated with presence of plaques in model 1 , and the association remained significant after adjusting for all covariates in model 3. If individuals with histories of stroke or cardiac diseases were included in the analyses, this association disappeared.

\section{DISCUSSION}

In the present study, we examined the association of flexibility with subclinical atherosclerosis in middle-aged men. Interestingly, our findings suggested that poor flexibility of the upper extremity was positively associated with IMT, while poor trunk flexibility was associated with presence of plaques in the common carotid artery. These associations remained significant even after adjusting for age, blood pressure, blood lipids, abdominal fat accumulation, handgrip strength and lifestyles. Because the arm extensibility and sit-and-reach tests are simple, easy, safe and inexpensive methods for evaluating physical fitness, we propose that poor flexibility may be a useful marker for subclinical atherosclerosis.

Previous studies have reported an association of arterial stiffness with poor trunk flexibility in middle-aged men (40-59 years), ${ }^{1011}$ which may support our findings as arterial stiffness is linked with arterial remodelling. Increased 
Table 1 Characteristics of participants according to arm extensibility and presence of common carotid plaque

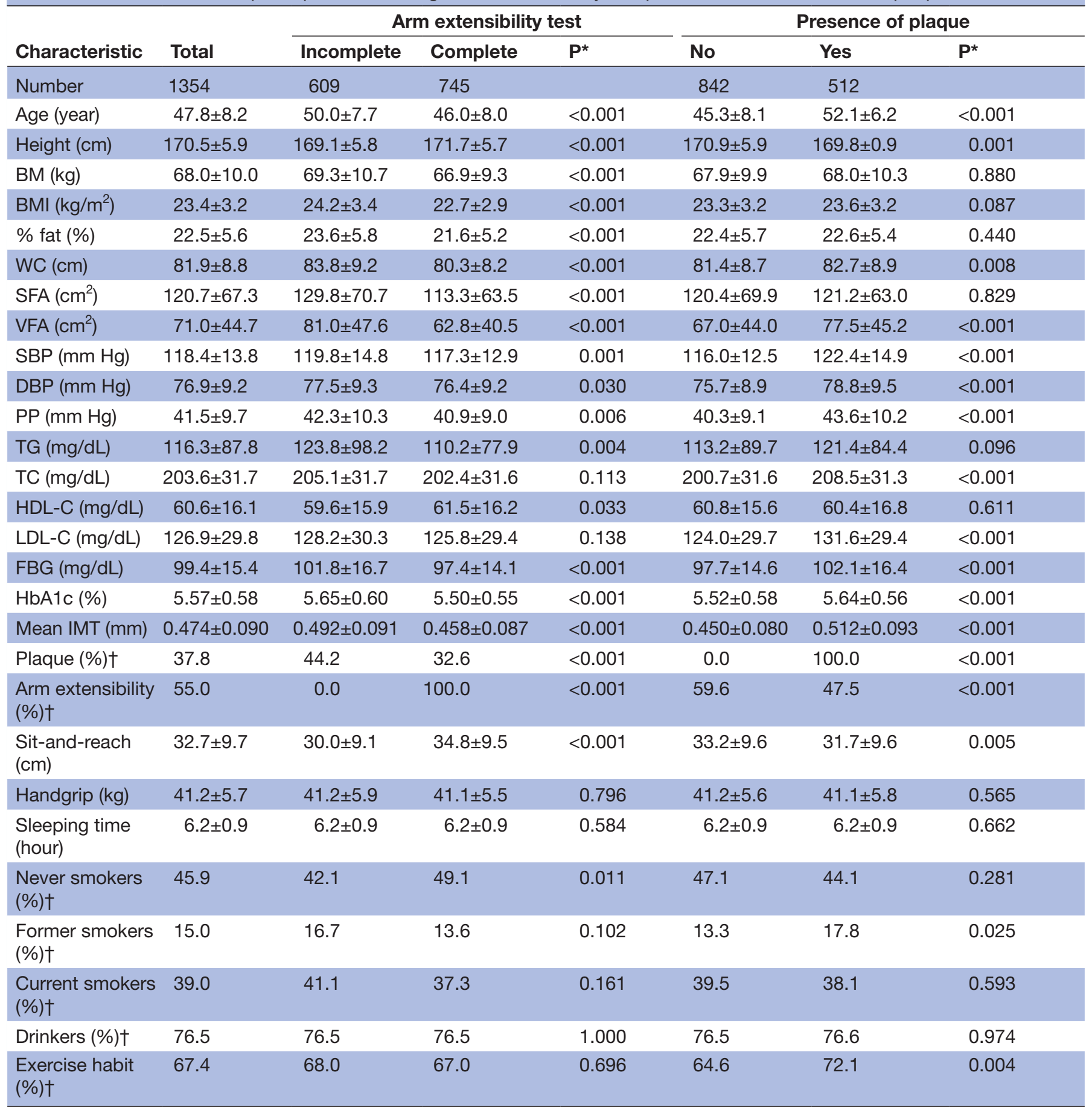

Data are mean \pm SD.

${ }^{*} \mathrm{P}$ values from unpaired $\mathrm{t}$ - test or $\chi^{2}$ test

$+\chi^{2}$ test.

BM, body mass; BMI, body mass index; \% fat, percentage of body fat; DBP, diastolic blood pressure; FBG, fasting blood glucose; HbA1c, glycated haemoglobin; HDL-C, high-density lipoprotein cholesterol; IMT, intima-media thickness; LDL-C, low-density lipoprotein cholesterol; PP, pulse pressure; SBP, systolic blood pressure; SFA, subcutaneous fat area; TC, total cholesterol; TG, triglyceride; VFA, visceral fat area; WC, waist circumference.

carotid-femoral PWV is also associated with progression of IMT and plaque formation in the carotid artery. ${ }^{16}$ Furthermore, the CAVI score is significantly correlated with carotid $\mathrm{IMT}^{12}$ and plaque formation. ${ }^{17}$ Atherosclerosis is an inflammatory process related to thickening of the arterial walls and plaque formation, which are, at least in part, accompanied by increased atheroma and smooth muscle cells in the intima, increased collagen and decreased elastin in the media, and deposition of calcium and advanced glycation end-products. ${ }^{18}$ These 
Table 2 Relationships between common carotid intimamedia thickness and arm extensibility or sit-and-reach tests using multivariate linear regression models

\begin{tabular}{|c|c|c|c|}
\hline & $\boldsymbol{\beta}$ & $95 \% \mathrm{Cl}$ & $\mathbf{P}$ \\
\hline \multicolumn{4}{|c|}{ Arm extensibility } \\
\hline Model $1^{\star}$ & -0.189 & -0.04382 to -0.02481 & $<0.001$ \\
\hline Model $2 \dagger$ & -0.085 & -0.02436 to -0.00655 & 0.001 \\
\hline Model 3‡ & -0.073 & -0.02224 to -0.00041 & 0.004 \\
\hline \multicolumn{4}{|c|}{ Sit-and-reach } \\
\hline Model 1* & -0.050 & -0.00097 to 0.00003 & 0.066 \\
\hline Model $2 \dagger$ & -0.006 & -0.00050 to 0.00111 & 0.323 \\
\hline Model 3‡ & 0.014 & -0.00032 to 0.00059 & 0.573 \\
\hline
\end{tabular}

${ }^{\star}$ Crude.

†Adjusted for age and height.

$\ddagger$ Adjusted for age, height, subcutaneous fat area, visceral fat area, diastolic blood pressure, pulse pressure, triglyceride, highdensity lipoprotein cholesterol, low-density lipoprotein cholesterol, fasting blood glucose, sleeping time, exercise habit, drinking habit, smoking habit, handgrip strength and sit-and-reach (arm extensibility analysis) or arm extensibility (sit-and-reach analysis).

characteristics can modify the mechanical properties of arteries. ${ }^{19}$

A potential explanation for the relationship between flexibility and arterial remodelling is that the exercises that improve flexibility may also regulate remodelling. Indeed, stretching and yoga exercise training can reduce arterial stiffness or enhance arterial compliance. ${ }^{20-22}$ Four weeks of static stretching training was reported to reduce the brachial-ankle PWV and CAVI score, and with improved sit-and-reach test performance, in middle-aged men. ${ }^{20}$ Furthermore, in older adults, stretching training for 13 weeks increased carotid artery compliance, while strength training or

Table 3 Relationships between arm extensibility or sitand-reach and presence of common carotid plaque using multivariate logistic regression models

\begin{tabular}{|c|c|c|c|}
\hline & OR & $95 \% \mathrm{Cl}$ & $\mathbf{P}$ \\
\hline \multicolumn{4}{|c|}{ Arm extensibility } \\
\hline Model $1^{*}$ & 0.61183 & 0.49029 to 0.76349 & $<0.001$ \\
\hline Model 2† & 0.92207 & 0.71812 to 1.18394 & 0.525 \\
\hline Model 3‡ & 0.97035 & 0.74309 to 1.26712 & 0.825 \\
\hline \multicolumn{4}{|c|}{ Sit-and-reach } \\
\hline Model $1^{*}$ & 0.98381 & 0.97260 to 0.99515 & 0.005 \\
\hline Model $2 \dagger$ & 0.99069 & 0.97841 to 1.00313 & 0.142 \\
\hline Model 3‡ & 0.98579 & 0.97257 to 0.99919 & 0.038 \\
\hline
\end{tabular}

${ }^{\star}$ Crude.

†Adjusted for age and height.

$\ddagger$ Adjusted for age, height, subcutaneous fat area, visceral fat area, diastolic blood pressure, pulse pressure, triglyceride, highdensity lipoprotein cholesterol, low-density lipoprotein cholesterol, fasting blood glucose, sleeping time, exercise habit, drinking habit, smoking habit, handgrip strength and sit-and-reach (arm extensibility analysis) or arm extensibility (sit-and-reach analysis). combination of strength and aerobic exercise training, did not. ${ }^{21}$ Similarly, in older subjects, 12 weeks of yoga training significantly reduced carotid-femoral PWV and brachial-ankle PWV while there was no effect of brisk walking training. ${ }^{22}$ It is likely that good flexibility is associated with low arterial stiffness and possible prevention of atherosclerosis, at least in part, via habitual exercises that can modify flexibility. Although our study design did not allow us to determine the exact cause, we found a potential role of exercises that improve flexibility on arterial remodelling, which partially affected the associations observed in this study.

The current study showed that arm extensibility predicted IMT, but not the presence of plaques, while the sit-and-reach test predicted the presence of plaques, but not IMT. The causes of these contrasting findings are unclear, although differences in the biological characteristics of the IMT and plaques may be important. The IMT consists of $\sim 80 \%$ media (smooth muscle cells) and $\sim 20 \%$ intima, whereas plaque formation is largely an intimal process composed of deposition of cholesterol and infiltration of inflammatory cells. ${ }^{23}$ IMT mainly reflects hypertensive hypertrophy of medial cells at early stages of atherosclerosis, while plaques reflect inflammation, oxidation, endothelial dysfunction and smooth muscle cell proliferation. ${ }^{23}$ In addition, genetic polymorphisms determining IMT are inconsistent with those determining plaque formation. ${ }^{24}$

Health-related physical fitness is composed of cardiorespiratory fitness, body composition, muscular strength and endurance, and flexibility. ${ }^{25}$ For muscular strength, we provide novel data that handgrip strength is not associated with IMT or plaque formation. Nevertheless, these findings do not rule out an association of muscular strength with atherosclerosis, as we did not estimate the strength of other muscle groups. Indeed, leg extension strength is inversely associated with brachial-ankle PWV, ${ }^{11}$ suggesting that reduced lower extremity muscular strength may predict atherosclerosis. Cardiorespiratory fitness is also inversely associated with carotid IMT in older adults ( $\geq 60$ years) ${ }^{26}$ Furthermore, good cardiorespiratory fitness is associated with slower progression of carotid arterial remodelling in middle-aged men (42-60 years). ${ }^{27}$ It remains unknown whether the association of carotid atherosclerosis with flexibility observed in the present study is mediated by other physical fitness variables. However, it was reported the association of sit-and-reach performance with brachial-ankle PWV is independent of cardiorespiratory fitness and leg strength. ${ }^{11}$ Thus, it is likely that the association of flexibility with carotid subclinical atherosclerosis is independent of cardiorespiratory fitness and muscular strength.

This study has four main limitations with respect to generalisability and interpretation of results. Because of its cross-sectional design, we were unable to determine the exact causes of the various relationships in our study. Future longitudinal studies are required to 
determine the causal relationship between flexibility and atherosclerosis. Second, the characteristics of the subjects in this study limited the generalisability of our results. All participants were middle-aged, male Japanese employees, and our findings cannot be applied to subjects of other ages, genders or ethnicities. Third, this study estimated only two types of flexibility. The arm extensibility test can evaluate the range of shoulder external rotation and forearm supination, while the sit-and-reach test can assess hamstring and lower back flexibility. However, the association of flexibility of other joints or muscle groups with atherosclerosis remains unknown. Fourth, we did not measure any indicators of arterial stiffness such as PWV, CAVI or $\beta$ stiffness. Therefore, the associations between body flexibility, atherosclerosis and arterial stiffness remain unclear. Instead, the associations of subclinical atherosclerosis indicators with body flexibility were adjusted by pulse pressure, which may partially reflect stiffened aorta that can increase SBP and decrease DBP through a variety of mechanisms. ${ }^{28-30}$

In summary, this cross-sectional study investigated the association of flexibility with subclinical atherosclerosis in Japanese middle-aged men. Poor arm extensibility was associated with increased common carotid IMT, while poor sit-and-reach performance was associated with carotid plaque formation. These associations were independent of age, blood pressure, blood lipids, abdominal fat accumulation, handgrip strength and lifestyle, including sleeping, drinking, exercise and smoking habits. Thus, poor flexibility may be a useful indicator of physical fitness for arterial remodelling. Future studies are required to evaluate the causal relationships, biochemical mechanisms and flexibility of other joints/muscle groups.

Acknowledgements The authors thank the participants and staff of the Exercise Support and WELPO Promotion Groups in the Health Support Center WELPO at Toyota Motor Corporation.

Contributors MS led the writing and data analysis. TI led data arrangement and participated in data collection and analysis. AK participated in data collection and arrangement. Ml critically reviewed the manuscript. TY led data collection and critically reviewed the manuscript. KK provided the study design and critically reviewed the manuscript. The authors declare that the results of the present study are presented clearly, honestly and without fabrication, falsification or inappropriate data manipulation.

Competing interests None declared.

Patient consent Obtained.

Ethics approval The study protocol was approved by the Ethics Committee of Toyota Memorial Hospital (Aichi, Japan).

Provenance and peer review Not commissioned; externally peer reviewed.

Data sharing statement The raw data cannot be made freely available in order to protect privacy and confidentiality, which does not allow public sharing of data on individuals. However, aggregated data are available on request. Requests should be sent to the corresponding author (MS) (masataka_suwa@mail.toyota. co.jp).

Open Access This is an Open Access article distributed in accordance with the Creative Commons Attribution Non Commercial (CC BY-NC 4.0) license, which permits others to distribute, remix, adapt, build upon this work non-commercially, and license their derivative works on different terms, provided the original work is properly cited and the use is non-commercial. See: http://creativecommons.org/ licenses/by-nc/4.0/

(C) Article author(s) (or their employer(s) unless otherwise stated in the text of the article) 2018. All rights reserved. No commercial use is permitted unless otherwise expressly granted.

\section{REFERENCES}

1. Bartels S, Franco AR, Rundek T. Carotid intima-media thickness (cIMT) and plaque from risk assessment and clinical use to genetic discoveries. Perspectives in Medicine 2012;1:139-45.

2. Lorenz MW, Markus HS, Bots ML, et al. Prediction of clinical cardiovascular events with carotid intima-media thickness: a systematic review and meta-analysis. Circulation 2007;115:459-67.

3. Polak JF, Pencina MJ, Pencina KM, et al. Carotid-wall intimamedia thickness and cardiovascular events. N Engl J Med 2011;365:213-21.

4. Rundek T, Arif H, Boden-Albala B, et al. Carotid plaque, a subclinical precursor of vascular events: the Northern Manhattan Study. Neurology 2008;70:1200-7.

5. Mathiesen EB, Johnsen SH. Ultrasonographic measurements of subclinical carotid atherosclerosis in prediction of ischemic stroke. Acta Neurol Scand 2009;120:68-72.

6. Spence JD. Measurement of intima-media thickness vs. carotid plaque: uses in patient care, genetic research and evaluation of new therapies. Int J Stroke 2006;1:216-21.

7. Leong DP, Teo KK, Rangarajan S, et al. Prognostic value of grip strength: findings from the Prospective Urban Rural Epidemiology (PURE) study. Lancet 2015;386:266-73.

8. Ross R, Blair SN, Arena R, et al. Importance of assessing cardiorespiratory fitness in clinical practice: a case for fitness as a clinical vital sign: a scientific statement from the american heart association. Circulation 2016;134:e653-699.

9. American College of Sports Medicine. Section 2, Exercise testing. 4, Health-related physical fitness testing and interpretation. In: Pescatello LS, Arena R, Riebe D, Thompson PD, et al. eds. ACSM's guidelines for exercise testing and prescription. 9th edn. Philadelphia (PA): Lippincott Williams \& Wilkins, 2014:60-113.

10. Nishiwaki M, Kurobe K, Kiuchi A, et al. Sex differences in flexibilityarterial stiffness relationship and its application for diagnosis of arterial stiffening: a cross-sectional observational study. PLoS One 2014;9:e113646.

11. Yamamoto $\mathrm{K}$, Kawano $\mathrm{H}$, Gando $\mathrm{Y}$, et al. Poor trunk flexibility is associated with arterial stiffening. Am J Physiol Heart Circ Physiol 2009;297:H1314-H1318.

12. Gomez-Sanchez L, Garcia-Ortiz L, Patino-Alonso MC, et al. The association between the cardio-ankle vascular index and other parameters of vascular structure and function in caucasian adults: MARK study. J Atheroscler Thromb 2015;22:901-11.

13. Suwa M, Imoto T, Kida A, et al. Age-related reduction and independent predictors of toe flexor strength in middle-aged men. J Foot Ankle Res 2017;10:15.

14. Anon. terminology and diagnostic criteria committee, Japan society of ultrasonics in medicine. standard method for ultrasound evaluation of carotid artery lesions. J Med Ultrason 2009;36:219-26.

15. Hiuge-Shimizu A, Kishida K, Funahashi T, et al. Absolute value of visceral fat area measured on computed tomography scans and obesity-related cardiovascular risk factors in large-scale Japanese general population (the VACATION-J study). Ann Med 2012;44:82-92.

16. van Popele NM, Grobbee DE, Bots ML, et al. Association between arterial stiffness and atherosclerosis: the Rotterdam Study. Stroke 2001;32:454-60.

17. Nakamura K, Tomaru T, Yamamura S, et al. Cardio-ankle vascular index is a candidate predictor of coronary atherosclerosis. Circ $J$ 2008;72:598-604.

18. Lee HY, Oh BH. Aging and arterial stiffness. Circ J 2010;74:2257-62.

19. Jani B, Rajkumar C. Ageing and vascular ageing. Postgrad Med J 2006;82:357-62.

20. Nishiwaki M, Yonemura $\mathrm{H}$, Kurobe $\mathrm{K}$, et al. Four weeks of regular static stretching reduces arterial stiffness in middle-aged men. Springerplus 2015;4:555.

21. Cortez-Cooper MY, Anton MM, Devan AE, et al. The effects of strength training on central arterial compliance in middle-aged and older adults. Eur J Cardiovasc Prev Rehabil 2008;15:149-55.

22. Patil SG, Aithala MR, Das KK. Effect of yoga on arterial stiffness in elderly subjects with increased pulse pressure: a randomized controlled study. Complement Ther Med 2015;23:562-9. 
23. Roquer J, Segura T, Serena J, et al. Value of carotid intima-media thickness and significant carotid stenosis as markers of stroke recurrence. Stroke 2011;42:3099-104.

24. Pollex RL, Hegele R. Genetic determinants of carotid ultrasound traits. Curr Atheroscler Rep 2006;8:206-15.

25. American College of Sports Medicine. Section 1, Health appraisal and risk assessment. 1, Benefits and risks associated with physical activity. In: Pescatello LS, Arena R, Riebe D, Thompson PD, et al. eds. ACSM's guidelines for exercise testing and prescription. 9th edn. Philadelphia (PA): Lippincott Williams \& Wilkins, 2014:2-18.

26. Gando Y, Yamamoto K, Kawano H, et al. Attenuated agerelated carotid arterial remodeling in adults with a high level of cardiorespiratory fitness. J Atheroscler Thromb 2011;18:248-54.

27. Lakka TA, Laukkanen JA, Rauramaa R, et al. Cardiorespiratory fitness and the progression of carotid atherosclerosis in middle-aged men. Ann Intern Med 2001;134:12-20.

28. Safar ME. Pulse pressure in essential hypertension: clinical and therapeutical implications. J Hypertens 1989;7:769-76.

29. Kelly RP, Tunin R, Kass DA. Effect of reduced aortic compliance on cardiac efficiency and contractile function of in situ canine left ventricle. Circ Res 1992;71:490-502.

30. Nichols WV, O'Rourke. McDonald's blood flow in arteries theoretic, experimental, and clinical principles. 3rd edn. London/Melbourne: $\mathrm{E}$ Arnold, 1990;142:77-142. 